\title{
Molecular recognition in supramolecular systems of humic substances
}

Jorobekova Sh.

Institute of Chemistry and Phytotechnologies, National Academy of Sciences, Bishkek, Kyrgyzstan; jorobekova@mail.ru

Keywords: humic substances, ligands, dissociation constants, molecular recognition

doi: 10.36291/HIT.2019.jorobekova.030

The key processes in biology and medicine are molecular recognition. In these processes, there is the formation of non-covalent complexes due to ionic, van der Waals, hydrogen and coordination bonds, as well as hydrophilic and hydrophobic interactions. The strength of the non-covalent interaction between the two molecules is characterized by the dissociation constant $\mathrm{Kd}$, which is related to the standard free energy by the equation $\Delta \mathrm{G}_{\text {free }}^{\circ}=\mathrm{RT}$ In $\mathrm{Kd}$. Humic substances (HS) as supramolecular receptors bind various ligands (metal ions, hydrophobic organic compounds, cationic and anionic polyelectrolytes, etc.) with the formation of non-covalent complexes characterized by the corresponding values of dissociation constants. In particular, according to the results of our studies, the Kd for humic acids (HA) with various metal ions vary from $10^{-3} \mathrm{M}$ to $10^{-10}$ $M$ in dependence on the reaction conditions. Complexes of HA with hydrophobic organic compounds are characterized by $\mathrm{Kd}$ values from $10^{-3} \mathrm{M}$ to $10^{-5} \mathrm{M}$. The strength of interpolyelectrolyte complexes (IPEC) is expressed by $\mathrm{Kd}$ values from $10^{-1} \mathrm{M}$ to $10^{-6} \mathrm{M}$. Corresponding to these $\mathrm{Kd}$ values, the free binding energy also changes. A characteristic feature of the molecular recognition by the humic receptors of the abovementioned ligands is their low specificity that can be explained by the dissipative nature of HS. Moreover, HS belong to systems with disordered bonds of elements that freely change their relationship to each other under various factors influence.

The conformational variability of HS has a great influence on the affinity for ligands. If necessary, part of the internal free energy of the HS goes to the establishment of conformation favorable for ligand binding and then $\Delta G_{\text {free }}$ decreases. Of particular importance are the hydrophilic/hydrophobic properties of HS. As a rule, the attachment of polar ligands to the HS occurs with the formation of the same hydrogen bonds that exist in the HS molecule. Hydrogen binding is a very important determinant of the specificity of molecular recognition. But, hydrogen binding does not always affect the change of free energy, since the energy that increases upon complexation represents a balance between the energy of hydrogen bonding in the complexes and the energy of cleavage of hydrogen bonds with the polar groups' ligands and HS. Hydrophobic compounds bind to HS differently depending on difference in their structure and provide a more accurate determination of the free binding energy in intermolecular interactions. 